\title{
Stability for a Class of Bilocal Hamiltonians *
}

\author{
Arthur Jaffe ${ }^{1,2}$, Andrzej Lesniewski ${ }^{1}$, and Konrad Osterwalder \\ Department of Mathematics, E.T.H. Zentrum, CH-8092 Zürich, Switzerland
}

Received September 4, 1992

Dedicated to Huzihiro Araki

\begin{abstract}
We introduce a method to establish stability of non-local interactions, and we apply this method to certain polynomial non-linear field theory. The non-local potential must satisfy the property of slow decrease at infinity in Fourier space (SDI).
\end{abstract}

\section{Introduction}

We extend the stability proof of constructive quantum field theory to a certain class of non-local Hamiltonians. Energy expressions for which the highest degree terms are non-local do not fit into the conventional proofs of stability. Our method is to show that a certain class of non-local interactions can be bounded from below by a local interaction - with a coupling constant that is "momentum dependent," and vanishes for large momentum. This method can be combined with an estimate on the measure of configurations for which the interaction is negative to establish -stability.

Non-local potentials of the form

$$
W=\int \mathscr{P}(x) V(x-y) \mathscr{P}(y) d x d y
$$

often occur in the physics literature, and it is useful to have methods to study them. One such example is the study of long range (infra-red) interactions. Another example arises from the introduction of a non-local cutoff in supersymmetric quantum field theories, which gives rise to interactions of the form (1.1). They are not amenable to the standard methods.

In (1.1) we consider polynomials $\mathscr{P}(x)$ in the field operator; furthermore we normal order (1.1), which we denote by $: W:$. The method used here works for

\footnotetext{
* Supported in part by the National Science Foundation under Grant PHY91-20626

1 Present Address: Harvard University, Cambridge, Massachusetts 02138, U.S.A.

2 John S. Guggenheim Foundation Fellow
} 
a special class of potentials $V$, namely those which have slow decrease at infinity in their Fourier transform (momentum space). This corresponds in constructive quantum field theory to a restricted class of cutoff functions. It is interesting that certain analytical methods in constructive quantum field theory are suited to a very restricted class of cutoffs. This differs from more standard methods which are not so closely tied to the analytical form of mollifiers.

We also investigate local and non-local perturbations of potentials (1.1) by polynomials $Q$ of degree less than twice the degree of $\mathscr{P}$, and we study the continuity of the corresponding heat kernels as a function of a parameter.

The interaction operator (1.1) is a multiplication operator on Fock space by the function $W$, namely by a polynomial $W(\cdot)$ in an infinite number of variables. These variables are the Fourier coefficients $\tilde{\varphi}(k), k \in \mathbb{Z}$ of $\varphi$. While the polynomial $W$ is unbounded from below, the operator $H_{0}+: W$ : is bounded from below. This lower bound can only be established for a certain class of cutoff functions in $W$.

\section{Slow Decrease at Infinity (SDI)}

On the circle of length $\ell$ (which we denote $S_{\ell}^{1}$ ) a function $\chi(x)$ can be defined by its Fourier series

$$
\chi(x)=\frac{1}{\ell} \sum_{p \in \tilde{S}} \tilde{\chi}(p) e^{-i p x}, \quad \tilde{\chi}(p)=\int \chi(x) e^{i p x} d x .
$$

We are interested in specifying $\chi$ by the Fourier coefficients $\tilde{\chi}$, and we consider that (2.1) converges in the sense of distributions. The Fourier coefficients define a multiplication operator $\tilde{\chi}$ on $l^{2}(\tilde{S})$, namely

$$
\tilde{\chi}: \tilde{f}(p) \rightarrow \tilde{\chi}(p) \tilde{f}(p), \quad \text { with norm } \quad\|\tilde{\chi}\|_{l^{2} \rightarrow l^{2}}=\sup _{p \in \tilde{S}}|\tilde{\chi}(p)| .
$$

By unitarity of the Fourier transformation from $L^{2}\left(S_{\ell}^{1}\right)$ to $l^{2}(\tilde{S})$ the convolution kernel $\chi(x-y)$ defines the corresponding operator on $L^{2}\left(S_{\ell}^{1}\right)$,

$$
\chi: f(\cdot) \rightarrow(\chi * f)(\cdot)=\int_{S_{\ell}^{1}} \chi(\cdot-y) f(y) d y,
$$

with norm

$$
\|\chi\|_{L^{2} \rightarrow L^{2}}=\|\tilde{\chi}\|_{l^{2} \rightarrow l^{2}} \text {. }
$$

We call $\chi$ (or $\tilde{\chi})$ a cutoff function if $\tilde{\chi}$ has three basic properties:

$$
\begin{gathered}
0 \leqq \tilde{\chi}(p) \leqq 1, \\
\tilde{\chi}(p)=\tilde{\chi}(-p), \\
\tilde{\chi}(0)=1 .
\end{gathered}
$$

As a consequence of (i), the operators $(2.2-3)$ defined for a cutoff $\chi$ are positive contractions. As a consequence of (iii), their norm is 1 . As a consequence of (ii), the kernel $\chi(x-y)$ is real, so the operator $\chi$ in (2.3) is real.

The class of cutoff functions includes characteristic functions in Fourier space, namely

$$
\tilde{\chi}_{\kappa}(p)= \begin{cases}1, & |p| \leqq \kappa \\ 0, & |p|>\kappa\end{cases}
$$


For shorthand, we reserve the subscript $\kappa$ to denote the action of the sharp cutoff function, namely

$$
\tilde{f}_{\kappa}(p)=\tilde{\chi}_{\kappa}(p) \tilde{f}(p) .
$$

In this note the key property of a cutoff is slow decrease at infinity (SDI). We are interested in restricted classes of cutoffs which do not include the sharp cutoff (II.6), but the sharp cutoff will be obtained as a limit of a sequence of cutoffs in these classes. Given $\varepsilon \geqq 0$, define the $\mathrm{SDI}_{\varepsilon}$ property by that

(iv) $\left(\mathrm{SDI}_{\varepsilon}\right)$. The cutoff $\chi$ is $\mathrm{SDI}_{\varepsilon}$ if there exist positive constants $\gamma, \delta, M$ such

$$
(1+\delta|p|)^{-\varepsilon} \leqq \tilde{\chi}(p) \leqq M(1+\gamma|p|)^{-\varepsilon} .
$$

The identity cutoff function $\tilde{\chi}(p) \equiv 1$ corresponds to the limiting case $\varepsilon=0$ or $\gamma=\delta=0, M=1$. Thus one can think of the $\mathrm{SDI}_{\varepsilon}$ property as a measure of the small deviation of $\tilde{\chi}(p)$ from the identity cutoff.

It is also possible to introduce a logarithmic SDI property, in which the bound (2.8) is replaced by a lower (and related upper) bound on the form

$$
(1+\delta|p|)^{-\varepsilon}(\ln 2)^{\alpha}(\ln (2+|p|))^{-\alpha} .
$$

The case $\varepsilon=0, \alpha>1$ can be used, for example, in certain supersymmetric quantum field theories.

In Sect. 5 we wish to define a family of cutoff functions $\chi_{\rho}$ which depend on a positive parameter $\rho$. This parameter we wish to interpret as a scaling parameter. In order to define $\chi_{\rho}$, we interpolate $\tilde{\chi}(p)$, defined for $p \in \mathbb{Z}$ to a function defined for all $p \in \mathbb{R}$. We choose a $C^{\infty}$ interpolation for $p \in \mathbb{R}$, which also satisfies $(2.5,2.8)$ for all $p \in \mathbb{R}$. We denote this interpolation also by $\tilde{\chi}(p)$. Clearly every cutoff satisfying $(2.5,2.8)$ has a $C^{\infty}$ interpolation which also satisfies $(2.5-8)$. While this interpolation is not unique, we can work with any choice of $C^{\infty}$ extrapolation. We then define

$$
\tilde{\chi}_{\rho}(p)=\tilde{\chi}(p / \rho)
$$

and

$$
\chi_{\rho}(x)=\frac{1}{\ell} \sum_{p \in \tilde{S}} \tilde{\chi}(p / \rho) e^{-i p x} .
$$

Given an $\mathrm{SDI}_{\varepsilon}$ cutoff $\chi$, the family $\chi_{\rho}$ is also $\mathrm{SDI}_{\varepsilon}$. In fact if $\tilde{\chi}$ satisfies (2.8), then

$$
(1+\delta|p| / \rho)^{-\varepsilon} \leqq \tilde{\chi}_{\rho}(p) \leqq M(1+\gamma|p| / \rho)^{-\varepsilon} .
$$

In other words the constants $\gamma, \delta$ are replaced by $\gamma / \rho, \delta / \rho$.

The $\rho \rightarrow \infty$ limit of any $\mathrm{SDI}_{\varepsilon}$ family $\chi_{\rho}$ is

$$
\lim _{\rho \rightarrow \infty} \tilde{\chi}_{\rho}(p)=1 \text {, }
$$

the identity cutoff. The $\mathrm{SDI}_{\varepsilon}$ property also gives an estimate on the rate at which the limits (2.12) is achieved. In particular, as $\rho \rightarrow \infty$,

$$
0 \leqq \tilde{\chi}_{\infty}(p)-\tilde{\chi}_{\rho}(p)=1-\tilde{\chi}_{\rho}(p) \leqq \varepsilon \delta|p| / \rho .
$$




\section{Stability}

Let $\varphi(x)$ denote a single-component, real, massive field defined on a bosonic Fock space $\mathfrak{F}$ over a circle of length $\ell$. The fundamental operators on $\mathfrak{F}$ are creation and annihilation operators $a(p)^{*}, a(p)$ defined for $p \in \tilde{S}$, and which satisfy

$$
\left[a(p), a^{*}\left(p^{\prime}\right)\right]=\delta_{p, p^{\prime}} I, \quad\left[a(p), a\left(p^{\prime}\right)\right]=0 .
$$

The Fock vacuum $\Omega_{0}$ is in the null space of all the $a(p)$, and the field $\varphi$ is

$$
\varphi(x)=\frac{1}{(2 \ell)^{1 / 2}} \sum_{p \in \tilde{S}} \mu(p)^{-1 / 2}\left(a(p)^{*}+a(-p)\right) e^{-i p x},
$$

where $\mu(p)=\left(p^{2}+m^{2}\right)^{1 / 2}$. The free field Hamiltonian is

$$
H_{0}=\sum_{p \in \tilde{S}} a(p)^{*} a(p) \mu(p) \text {. }
$$

Normal ordering is denoted by : : and is defined as a linear operator on polynomials in $a$ 's and $a^{*}$ 's; a normal ordered monomial of $a$ 's and $a^{*}$ 's has the $a^{*}$ 's acting after the $a$ 's. Let

$$
\mathscr{P}(\xi)=\sum_{j=0}^{n} \alpha_{j} \xi^{j}
$$

be a complex polynomial of degree $n>0$. Let $\mathscr{P}(\xi)^{-}$denote the complex conjugate polynomial.

We define a multiplication operator $W$ on $\mathfrak{F}$ by the expression

$$
W^{\chi}=W\left(\varphi_{\chi}\right)=\int_{S_{\ell}^{1} \times S_{\ell}^{1}}: \overline{\mathscr{P}\left(\varphi_{\chi}(x)\right)} V(x-y) \mathscr{P}\left(\varphi_{\chi}(y)\right): d x d y,
$$

where $V(x)$ is an $\operatorname{SDI}_{\varepsilon}$ function. Here $\varphi_{\chi}=\chi * \varphi$ is the field $\varphi$ smoothed with a cutoff function satisfying (2.5). (The function $\tilde{\chi}(p)$ is assumed to satisfy (2.5), but this function may or may not be $\mathrm{SDI}_{\varepsilon}$. In particular, it may also be the identity cutoff $\tilde{\chi}(p)=1$.) We let $W_{\kappa}^{\chi}$ denote the expression (3.3) with $\varphi_{\chi}$ replaced by $\varphi_{\chi \kappa}$, namely by the sharp cutoff (2.6) applied to $\varphi_{\chi}$. This has Fourier coefficients

$$
\tilde{\varphi}_{\chi \kappa}(p)=\left\{\begin{array}{cl}
\tilde{\varphi}(p) \tilde{\chi}(p), & \text { if }|p| \leqq \kappa \\
0, & \text { if }|p|>\kappa
\end{array} .\right.
$$

Both $W^{\chi}$ and $W_{\kappa}^{\chi}$ are multiplication operators on $\mathfrak{F}$. By the standard methods of constructive quantum field theory, the domain of $W^{\chi}$ and $W_{\kappa}^{\chi}$ (including the cases $\tilde{\chi}(p) \equiv 1)$ contains $\mathscr{D}$, the set of all vectors in $\mathfrak{F}$ with a finite number of particles and wave functions with compact support in $p$-space. These operators are essentially self adjoint on $\mathscr{D}$.

Theorem 3.1. (Stability) Let $W^{\chi}, W_{\kappa}^{\chi}$ be defined as above, where $\chi$ satisfies (2.5) and where $V$ is $\mathrm{SDI}_{\varepsilon}$ with $0 \leqq \varepsilon \leqq 1 /\left(8 n^{2}\right)$. Then on the domain $\mathscr{D}$ the operators

$$
H^{\chi}=H_{0}+W^{\chi} \text { and } H_{\kappa}^{\chi}=H_{0}+W_{\kappa}^{\chi}
$$

are bounded from below uniformly in $\chi, \kappa$.

2. Furthermore for $T>0$,

$$
\operatorname{Tr}\left(e^{-T H^{\alpha}}\right)<\infty, \quad \operatorname{Tr}\left(e^{-T H_{\kappa}^{\alpha}}\right)<\infty,
$$

with the traces bounded uniformly in $\chi, \kappa$. 
Remarks. Without the normal ordering, $W$ would be a positive sesquilinear form. In the case that $\chi$ decreases sufficiently fast at infinity, then $W$ extends to an unbounded multiplication operator. However the spectrum of $W$ in general covers the real line. In case $W$ is normal ordered, then $W$ extends to a densely defined operator even though $\chi$ may not tend to 0 .

In spite of the fact that $W$ is unbounded from below, the operator $H^{\chi}$ is bounded from below. Its stability is restored because $H_{0}$ grows sufficiently on vectors where $W$ is very negative. This requires that $V$ satisfy the $\mathrm{SDI}_{\varepsilon}$ property for $\varepsilon$ sufficiently small.

In case $\varepsilon=0$ or $\tilde{\chi}=1$, the stability of $H^{\chi}$ is a classic result of constructive quantum field theory [3], see also [1]. However, in the case $\varepsilon>0$, the Hamiltonian $H$ is essentially non-local. In that case, stability of $H$ is a new result; it is even new for the case of fixed $\kappa<\infty$.

The nontrivial nature of this result can be seen by considering

$$
W_{\kappa}^{\chi}=:\left(\int_{S_{f}^{1}} \varphi_{\chi}(x)^{3} d x\right)^{2}:,
$$

which is of the form considered, except $\tilde{V} \equiv 1$ so that $V$ is not $\mathrm{SDI}_{\varepsilon}$. In this case $\int \varphi_{\chi}^{3} d x$ can be small on vectors for which $\int \varphi_{\chi}^{2} d x$ is large. Analysis of this effect leads to the conclusion that for certain functions $\chi$, the operator $H_{0}+W_{\kappa}^{\chi}$ is unbounded from below! Even for three degrees of freedom, namely for the choice

$$
\tilde{\chi}(p)= \begin{cases}1, & \text { if } p=0 \\ \delta, & \text { if }|p|=2 \pi / \ell \\ 0, & \text { if }|p|>2 \pi / \ell\end{cases}
$$

there is an unusual phenomenon. In this special case, the operator $H^{\chi}$ is bounded from below for each $0 \leqq \delta \leqq 1$. Surprisingly, the lower bound diverges to $-\infty$ as $\delta \rightarrow 0+$, and hence the lower bound is discontinuous at $\delta=0$. This example illustrates the robust nature of the SDI property in proving stability bounds.

In the following section we extend Theorem 3.1 and establish stability for perturbed Hamiltonians of the form $H_{\kappa}^{\chi}+Q_{\kappa}$, occasionally suppressing the superscript $\chi$. Here $Q_{\kappa}$ is a non-local polynomial in $\varphi$ of degree $2 n-1$ or less.

The novelty of this paper is the non-local nature of the interaction monomial of highest degree, which we bound from below by a local polynomial with a momentum dependent coupling constant. Other stability proofs (using for example phase cell or renormalization group methods) also rely on the leading order polynomial interaction to be local. Thus it is presumably interesting to incorporate the $\mathrm{SDI}_{\varepsilon}$ method introduced here into such arguments.

As a corollary of our method of proof, we can follow standard constructive quantum field theory analysis to establish:

Corollary 3.2. The operators $H_{\kappa}$ and $H$ are essentially self adjoint. Furthermore for $T>0$

$$
\lim _{\kappa \rightarrow \infty}\left\|e^{-T H_{\kappa}}-e^{-T H}\right\|_{\mathrm{HS}}=0,
$$

where HS denotes the Hilbert-Schmidt norm. 
Proof of Theorem 3.1. We introduce an auxiliary sharp cutoff $\kappa^{\prime}$ and field $\varphi_{\chi \kappa \kappa}$ with Fourier series

$$
\tilde{\varphi}_{\chi \kappa \kappa^{\prime}}(p)=\left\{\begin{array}{ll}
\tilde{\varphi}(p) \chi(p), & \text { if }|p| \leqq \min \left(\kappa, \kappa^{\prime}\right) \\
0, & \text { otherwise }
\end{array} .\right.
$$

If $\kappa<\infty$, then the auxiliary cutoff plays a role only for $\kappa>\kappa^{\prime}$. In what follows we omit explicit consideration of $\kappa$. For simplicity of notation we denote $\kappa^{\prime}$ by $\kappa$. The modification to include the analysis of both $\kappa$ and $\kappa^{\prime}$ (to obtain bounds which are uniform in $\kappa$ ) are a straightforward extension of the argument we present.

We prove that the functional integral representation for $\operatorname{Tr}(\exp (-T H))$ is bounded. The functional integral representation for the trace of the heat kernel is obtained by studying a perturbation of the Gaussian measure $d \mu_{C}$ of generalized functions $\mathscr{D}^{\prime}$ defined on the torus $\mathscr{T}$, where $\mathscr{T}=S_{\ell}^{1} \times S_{T}^{1}$ is the toroidal compactification of the cylindrical space-time $S_{\ell}^{1} \times \mathbb{R}$, see [2]. Let $\Delta$ denote the Laplacian on $\mathscr{T}$, and let $C=\left(-\Delta+m^{2}\right)^{-1}, m>0$. The measure $d \mu_{C}$ is the Gaussian on $\mathscr{D}^{\prime}(\mathscr{T})$ with mean zero, with covariance $C$, and with the normalization

$$
\int d \mu_{C}=\operatorname{Tr}\left(e^{-T H_{0}}\right) .
$$

The action functional $\mathscr{S}(\Phi)$ is defined as follows for $\Phi \in \mathscr{D}^{\prime}(\mathscr{T})$,

$$
\mathscr{S}^{\chi}=\mathscr{S}\left(\Phi_{\chi}\right)=\int_{S_{\ell}^{1} \times S_{\ell}^{1} \times S_{T}^{1}}: \overline{\mathscr{P}\left(\Phi_{\chi}(x, t)\right)} V(x-y) \mathscr{P}\left(\Phi_{\chi}(y, t)\right): d x d y d t .
$$

Here normal ordering of a monomial $\Phi(f)^{n}$ means to orthogonalize the monomials $\Phi(f)^{n}$ with respect to $d \mu_{C}$. Then the Feynman-Kac formula is the identity which relates the heat kernel of $H^{\chi}$ with $\mathscr{S}^{\chi}$,

$$
\operatorname{Tr}\left(e^{-T H^{x}}\right)=\int_{\mathscr{D}} e^{-\mathscr{T})} d \mu_{C}
$$

The field $\Phi_{\chi}$ in (3.7) is a classical variable, and its cutoffs correspond to $W^{\chi}$ in (3.3). In particular

$$
\Phi_{\chi}(x, t)=\int_{S_{\ell}^{1}} \chi(x-y) \Phi(y, t) d y .
$$

In other words the cutoff $\chi$ acts only on the spatial variable $x$,

$$
\Phi_{\chi}^{\sim}(p, t)=\tilde{\chi}(p) \int_{S_{\ell}^{1}} \Phi(x, t) e^{i p x} d x
$$

We also introduce $\Phi_{\chi \kappa}$ defined by its Fourier series in $x$,

$$
\Phi_{\chi}^{\sim}(p, t)_{\kappa}=\left\{\begin{array}{ll}
\Phi_{\chi}^{\sim}(p, t), & \text { for }|p| \leqq \kappa, \\
0, & \text { for }|p|>\kappa .
\end{array} .\right.
$$

We use $\Phi_{\chi \kappa}$ to construct $\mathscr{S}_{\kappa}^{\chi}$, namely $\mathscr{S}_{\kappa}^{\chi}=\mathscr{S}\left(\Phi_{\chi \kappa}\right)$. Then the corresponding Feynman-Kac identity is

$$
\operatorname{Tr}\left(e^{-T H_{\wedge}^{\chi}}\right)=\int_{\mathscr{D}^{\prime}(\mathscr{T})} e^{-S_{\varkappa}^{\chi}} d \mu_{C}
$$

As before, to simplify notation we at times suppress $\chi$.

In order to bound (3.8), we study

$$
f(a)=\mu_{C}\left\{e^{-s} \geqq e^{a+1}\right\},
$$


which has the property

$$
\int e^{-s} d \mu_{C}=\int_{-\infty}^{\infty} e^{a+1} f(a) d a
$$

Note that $f(-\infty)=\operatorname{Tr}\left(e^{-T H_{0}}\right)$ and that $f(a)$ is monotonically decreasing in $a$. Hence the integral (3.10) converges if and only if $f(a)$ tends to zero sufficiently rapidly so that $e^{a} f(a)$ is integrable. Because $f(a)$ is monotone, it is sufficient to show that

$$
\sup _{a>0} \mid a^{2} e^{a} f(a) \leqq \text { const. . }
$$

Proposition 3.3. There exist constants $\bar{\delta}$ and $\bar{\kappa}$ such that for $\delta \leqq \bar{\delta}$ and $\kappa \geqq \bar{\kappa}$,

$$
\mu_{C}\left\{\left|\mathscr{S}-\mathscr{S}_{\kappa}\right| \geqq 1\right\} \leqq e^{-\kappa^{1 / 4 n}} .
$$

Proposition 3.4. Let $P(\xi)$ be given as in (3.2), and let $\tilde{\varepsilon} \in[0,1)$ Then there exist constants $M, \bar{\delta}$, and $\bar{\kappa}$ such that for $\delta \leqq \bar{\delta}$ and $\kappa \geqq \bar{\kappa}$,

$$
\mathscr{S}_{\kappa} \geqq \tilde{\varepsilon}(1+n \delta \kappa)^{-\varepsilon}\left|\alpha_{n}\right|^{2}\left\|\Phi_{\kappa}\right\|_{L^{2 n}(\mathscr{T})}^{2 n}-M \kappa^{2 n \varepsilon} .
$$

Let us assume the propositions and derive inequality (3.11), thus completing the proof of the theorem. As a consequence of (3.13) we have for $\kappa \geqq \bar{\kappa}$,

$$
e^{-\mathscr{S}}=e^{-S_{\kappa}} e^{-\left(\mathscr{S}-\mathscr{S}_{\kappa}\right)} \leqq e^{M \kappa^{2 n \varepsilon}+1} e^{-1-\left(\mathscr{S}-\mathscr{S}_{\kappa}\right)} .
$$

Defining $a(\kappa)=M \kappa^{2 n \varepsilon}+1$, we see that

$$
e^{-\mathscr{S}} \leqq e^{a} e^{-1-\left(\mathscr{S}-\mathscr{I}_{n}\right)} .
$$

Thus whenever $e^{-\mathscr{S}} \geqq e^{a}$, it must be true that $\left|\mathscr{S}-\mathscr{S}_{\kappa}\right| \geqq 1$. In other words

$$
f(a(\kappa)) \leqq \mu_{C}\left\{\left|\mathscr{S}-\mathscr{S}_{\kappa}\right| \geqq 1\right\}
$$

Then by the assumed bound (3.12),

$$
f(a(\kappa)) \leqq e^{-\kappa^{1 / 4 n}}
$$

and for $\varepsilon<\left(8 n^{2}\right)^{-1}$, we have

$$
\left|a(\kappa)^{2} e^{a(\kappa)} f(a(\kappa))\right| \leqq\left(M \kappa^{2 n \varepsilon}+1\right)^{2} e^{\left(M \kappa^{2 n \varepsilon}+1\right)-\kappa^{1 / 4 n}} \leqq \text { const. }
$$

which is (3.11). This completes the reduction of Theorem 3.1 to Propositions 3.3-3.4.

Proof of Proposition 3.3. This bound is proved by a standard asymptotic method. See e.g. $[3,1]$ for more details. Briefly, for any $r>0$,

$$
\mu_{C}\left\{\left|\mathscr{S}-\mathscr{S}_{\kappa}\right| \geqq 1\right\} \leqq \int\left(\mathscr{S}-\mathscr{S}_{\kappa}\right)^{2 r} d \mu_{C} .
$$

For integer $r$, this polynomial moment of the Gaussian measure $d \mu_{C}$ reduces to a sum of $O(4 n r ! !)$ terms. Each term can be expressed as a sum or integral over a finite dimensional space, of kernels composed of products of $\tilde{C}(p), \tilde{V}(p), \tilde{\chi}(p)$, $\tilde{\chi}_{\kappa}(p)$, and the coefficients $\alpha_{j}$ of (3.2). Each such term can be bounded by the $L^{2}$ norm of its kernel. The difference $\mathscr{S}-\mathscr{S}_{\kappa}$ yields a kernel with convergent integral in the $L^{2}$ norm, having a convergence rate of $O\left(\kappa^{-\alpha}\right)$ for $\alpha<1 / 2$, as $\kappa \rightarrow \infty$. We use 
$\alpha=\frac{1}{4}$ to obtain the bound

$$
\mu_{C}\left\{\left|\mathscr{S}-\mathscr{S}_{\kappa}\right| \geqq 1\right\} \leqq M^{r} \kappa^{-r / 2} r^{2 n r},
$$

where $M$ is a constant depending on $n$, on the mass $m$, and on the constants $\alpha_{n}$, but it is bounded uniformly in $\tilde{\chi}$ and in $\tilde{V}$. This bound is optimized by $r=\kappa^{1 / 4 n}(1 / e M)^{1 / 2 n}$, yielding

$$
\mu_{\mathrm{C}}\left\{\left|\mathscr{S}-\mathscr{S}_{\kappa}\right| \geqq 1\right\} \leqq A e^{-B \kappa^{1 / 4 n}} .
$$

Choosing $\alpha$ slightly larger than $1 / 4$, we can replace $A$ and $B$ by 1 , thus proving (3.12).

Proof of Proposition 3.4. Our new idea is contained in the proof of this bound. It is sufficient to prove the proposition for the case $\alpha_{n}=1$, since for $\alpha \in \mathbb{C}$,

$$
\mathscr{S}_{\kappa}^{\alpha \mathscr{P}}=|\alpha|^{2} \mathscr{S}_{\kappa}^{\mathscr{P}} \text {. }
$$

If the inequality (3.13) holds with the constant $M$ for $\alpha_{n}=1$, then it holds for $\alpha_{n} \neq 1$ with the constant $M$ replaced by the constant $\left|\alpha_{n}\right|^{2} M$. We thus restrict attention to $\alpha_{n}=1$. In order to simplify notation, we first assume that $\mathscr{P}(\xi)=\xi^{n}$ is a monomial. We return at the end to establish the proposition for general $\mathscr{P}$. We start with the formula for : $\Phi_{\kappa}^{j}$,

$$
: \Phi_{\kappa}(x, t)^{j}:=\sum_{r=0}^{[j / 2]} c_{\kappa}^{r} \frac{j !}{(j-2 r) ! r ! 2 r}(-1)^{r} \Phi_{\kappa}(x, t)^{j-2 r} .
$$

Here $[j / 2]$ denotes the integer part of $j / 2$. Also $c_{\kappa} \equiv c_{\kappa}(0)$, where

$$
c_{\kappa}(x-y)=\int \Phi_{\kappa}(x, t) \Phi_{\kappa}(y, t) d \mu_{C}=\frac{1}{\ell} \sum_{|p| \leqq \kappa} \frac{1}{2 \mu(p)}|\tilde{\chi}(p)|^{2} e^{-i p(x-y)} .
$$

For some constant $M_{1}<\infty$,

$$
\left|c_{\kappa}(x-y)\right| \leqq c_{\kappa}(0)=c_{\kappa} \leqq M_{1} \ln (2+\kappa),
$$

with the constant $M_{1}$ uniform in $\chi$. The definition (3.16) extends by linearity to define : $\Phi_{\kappa}\left(x_{1}, t\right) \cdots \Phi_{\kappa}\left(x_{n}, t\right)$ : so we can write, in the case $\mathscr{P}(\xi)=\xi^{n}$,

$$
\mathscr{S}_{\kappa}=\sum_{j=0}^{n} \sum_{r, s \leqq[(n-j) / 2]} S_{r s j}(\kappa)
$$

where

$$
s_{r s j}(\kappa)=(-1)^{r+s+j} c_{\kappa}^{r+s} \alpha_{r s j} W(n-2 r-j, n-2 s-j, j) .
$$

Here $\alpha_{r s j}$ is the combinatorial coefficient

$$
\alpha_{r s j}=\left(\begin{array}{c}
n-j \\
2 r
\end{array}\right)\left(\begin{array}{c}
n-j \\
2 s
\end{array}\right)\left(\begin{array}{l}
n \\
j
\end{array}\right)^{2} j !(2 r-1) ! !(2 s-1) ! !,
$$

and

$$
W(k, l, j)=\int_{S_{\ell}^{1} \times S_{\ell}^{1} \times S_{T}^{1}} \Phi_{\kappa}(x, t)^{k} c_{\kappa}(x-y)^{j} V(x-y) \Phi_{\kappa}(y, t)^{l} d x d y d t .
$$

The constants $\alpha_{r s j}$ depend only on $n, r, s$ and $j$, and $\alpha_{000}=1$. 
The outline of the proof is to bound $s_{000}(\kappa)$ from below, and to use the estimate

$$
\sum_{r+s+j \neq 0}\left|s_{r s j}(\kappa)\right| \leqq \frac{1}{4}(n+1)^{3} \sup _{r+s+j \neq 0}\left|s_{r s j}(\kappa)\right|
$$

based on the number of $s_{r s j}(\kappa)$ 's to establish a lower bound on $\mathscr{S}_{\kappa}$, namely

$$
\mathscr{S}_{\kappa} \geqq s_{000}(\kappa)-\frac{1}{4}(n+1)^{3} \sup _{r+s+j \neq 0}\left|s_{r s j}(\kappa)\right| .
$$

Using (3.20), the proof of Proposition 3.4 for $\mathscr{P}(\xi)=\xi^{n}$ follows immediately from:

Lemma 3.5. For $\mathscr{S}_{\kappa}$ given in (3.18), with $\mathscr{P}(\xi)=\xi^{n}$, the following bounds hold:

$$
s_{000}(\kappa) \geqq(1+n \delta \kappa)^{-\varepsilon} \int_{\mathscr{T}} \Phi_{\kappa}(x, t)^{2 n} d x d t .
$$

(ii) For any $\varepsilon_{1}>0$, there exists $M<\infty$ such that for $r+s+j \neq 0$,

$$
\left|s_{r s j}(\kappa)\right| \leqq \varepsilon_{1}(1+n \delta \kappa)^{-\varepsilon} \int_{\mathscr{T}} \Phi_{\kappa}(x, t)^{2 n} d x d t+M\left((\delta \kappa)^{2 n \varepsilon}+1\right) \ln (2+\kappa)^{n} .
$$

Proof. The bound (i) is a consequence of the fact that $\Phi_{\kappa}(0, t)$ contains only Fourier modes with $|p| \leqq \kappa$, for $p \in \tilde{S}$. It follows that $\Phi_{\kappa}(x, t)^{n}$ contains only Fourier modes with $|p| \leqq n \kappa$. Thus we use the $\mathrm{SDI}_{\varepsilon}$ property to establish (3.21). Essentially the bound (3.21) says that the operator $V$ with kernel $V(x-y)$, when restricted to the subspace $L_{n \kappa}^{2}\left(S_{\ell}^{1}\right)$ spanned by Fourier modes with $|p| \leqq n \kappa$, is bounded from below by $(1+n \delta \kappa)^{-\varepsilon}$.

In order to prove (ii), we introduce some further notation. Let $T_{j}$ denote the operator on $L^{2}\left(S_{\ell}^{1}\right)$ with integral kernel

$$
T_{j}(x-y)=c_{\kappa}(x-y)^{j} V(x-y) .
$$

Then

$$
W(k, l, j)=\int_{S_{1}^{1}}\left\langle\Phi_{\kappa}^{k}, T_{j} \Phi_{\kappa}^{l}\right\rangle d t
$$

with $\langle\cdot, \cdot\rangle$ denoting the inner product on $L^{2}\left(S_{\ell}^{1}\right)$. We also let $\langle\cdot, \cdot\rangle_{L^{2}(\mathscr{T})}$ denote the inner product on $L^{2}$ of the torus. Thus

$$
W(k, l, j)=\left\langle\Phi_{\kappa}^{k},\left(T_{j} \otimes I\right) \Phi_{\kappa}^{l}\right\rangle_{L^{2}(\mathscr{T})} .
$$

We summarize some properties of the $W$ 's which are useful in this and in later sections.

Proposition 3.6. (i) The operators $T_{j}$ on $L^{2}\left(S_{\ell}^{1}\right)$ sastisfy

$$
0 \leqq T_{j} \leqq c_{\kappa}^{j} I
$$

where $c_{\kappa}$ is bounded in (3.17),

$$
\begin{gathered}
|W(k, l, j)| \leqq W(k, k, j)^{1 / 2} W(l, l, j)^{1 / 2} . \\
W(k, k, j) \leqq c_{\kappa}^{j}\left\|\Phi_{\kappa}\right\|_{L^{2 k}(\mathscr{T})}^{2 k} .
\end{gathered}
$$

(iv) Let $k, l \leqq n, \quad k+l \leqq 2 n-1$. Given $\varepsilon_{1}>0$, there exists $M<\infty$ such that

$$
\begin{aligned}
c_{\kappa}^{n-(k+l) / 2-j}|W(k, l, j)| \leqq & \varepsilon_{1}(1+n \delta \kappa)^{-\varepsilon}\left\|\Phi_{\kappa}\right\|_{L^{2 n}(\mathscr{T})}^{2 n} \\
& +M(1+n \kappa \delta)^{\varepsilon(2 n-1)} \ln (2+\kappa)^{n} .
\end{aligned}
$$


(v) If in (iii) $k+l \leqq 2 n-2$, then $(1+n \kappa \delta)^{\varepsilon(2 n-1)}$ in (3.28) can be replaced by $(1+n \delta \kappa)^{\varepsilon(n-1)}$.

(vi) Given $\bar{\delta}>0, \bar{\kappa}>0$, the second term in (3.28) can be replaced by $M \kappa^{2 n \varepsilon}$, uniformly in $0<\delta \leqq \bar{\delta}$, and $\kappa \geqq \bar{\kappa}$.

Proof. (i) As a convolution kernel, $T_{j}$ is a multiplication operator on momentum space. In fact, $\tilde{T}_{j}$ is the $j$-fold convolution

$$
\tilde{T}_{j}(p)=\left(\tilde{c}_{\kappa} * \tilde{c}_{\kappa} * \cdots * \tilde{c}_{\kappa} * \tilde{V}\right)(p)
$$

with

$$
\tilde{c}_{\kappa}(p)=\frac{1}{2 \mu(p)} \tilde{\chi}_{\kappa}(p)^{2} \geqq 0 .
$$

Since both $\tilde{c}(p)$ and $\tilde{V}(p)$ are positive, we infer $\tilde{T}_{j}(p) \geqq 0$. The upper bound on $\tilde{T}_{j}(p)$ follows from

$$
\left\|\tilde{T}_{j}(p)\right\|_{l^{\infty}(\tilde{S})} \leqq\left\|\tilde{c}_{\kappa}\right\|_{l^{1}(\tilde{S})}^{j}\|\tilde{V}\|_{l^{\infty}(\tilde{S})}=c_{\kappa}^{j} .
$$

(ii) This inequality is the Schwarz inequality applied to $W(k, l, j)$, and takes into account the fact that $T_{j} \geqq 0$ has a square root. We apply the Schwarz inequality on $L^{2}(\mathscr{T})$ to $(3.24)$, and use $\left(T_{j} \otimes I\right)^{1 / 2}=T_{j}^{1 / 2} \otimes I$.

(iii) Use (ii) and the upper bound

$$
T_{j} \otimes I \leqq c_{\kappa}^{j} \otimes I
$$

to establish (3.27).

(iv-v) Using (ii) and (iii), we infer

$$
c_{\kappa}^{n-(k+l) / 2-j}|W(k, l, j)| \leqq c_{\kappa}^{n-(k+l) / 2}\left\|\Phi_{\kappa}\right\|_{L^{2 k}(\mathscr{T})}^{k}\left\|\Phi_{\kappa}\right\|_{L^{2 l}(\mathscr{T})}^{l}
$$

We now analyze (3.33) in some detail. For simplicity we denote $c_{\kappa}$ by $c$. We consider two cases of the bound (3.33).

Case $k, l \leqq n-1$. In this case, we bound (3.33) by

$$
c^{n-(k+l) / 2-j}|W(k, l, j)| \leqq \sup _{u \in\{k, l\}}\left(c^{n-u}\left\|\Phi_{\kappa}\right\|_{L^{2 u}(\mathscr{T})}^{2 u}\right) .
$$

If the sup occurs for $u=0$, then we use bound (3.17) for $c$ to obtain

$$
c^{n-(k+l) / 2-j}|W(k, l, j)| \leqq c^{n} l T \leqq l T M_{1}^{n} \ln (2+\kappa)^{n} .
$$

The expression (3.35) is bounded by the right side of (3.28), with $\varepsilon_{1}=0$ and an appropriate constant $M$.

If the sup occurs for $1 \leqq u \leqq n-1$, then we use the elementary bound for $a, b>0$,

$$
a b \leqq \frac{1}{p} a^{p}+\frac{1}{q} b^{q},
$$

with $p, q$ dual exponents. Let $p=n / u$ and $q=n /(n-u)$, so $q / p=u /(n-u)$, and

$$
\frac{1}{(n-1)} \leqq \frac{q}{p} \leqq(n-1) \text {. }
$$


We apply the inequality to the right side of (3.34) with

$$
a=w \Phi_{\kappa}^{2 u}, \quad b=w^{-1} c^{n-u},
$$

where $\varepsilon_{1}<1$ and $w$ is the constant

$$
w=\varepsilon_{1}^{1 / p}(1+n \delta \kappa)^{-\varepsilon / p} .
$$

Then

$$
c^{n-u} \Phi_{\kappa}^{2 u} \leqq \varepsilon_{1}(1+n \delta \kappa)^{-\varepsilon} \Phi_{\kappa}^{2 n}+\left(\varepsilon_{1}^{-1} c\right)^{n}(1+n \delta \kappa)^{\varepsilon(n-1)},
$$

and so we have

$$
\begin{aligned}
c^{n-(k+l) / 2-j}|W(k, l, j)| \leqq & \varepsilon_{1}(1+n \delta \kappa)^{-\varepsilon}\left\|\Phi_{\kappa}\right\|_{L^{2 n}(\mathscr{T})}^{2 n} \\
& \times\left(\varepsilon_{1}^{-1} c\right)^{n}(1+n \delta \kappa)^{\varepsilon(n-1)} \ell T .
\end{aligned}
$$

Using (3.17) to bound $c^{n}$, we obtain a bound of the desired form (3.28).

Case $l \leqq n-1, k=n$. (The case $l=n, k \leqq n-1$ is similar.) We bound (3.33) by

$$
\begin{aligned}
c^{(n-l) / 2-j}|W(k, l, j)| \leqq & \frac{1}{2} \varepsilon_{1}(1+n \kappa \delta)^{-\varepsilon}\left\|\Phi_{\kappa}\right\|_{L^{2 n}(\mathscr{T})}^{2^{n}} \\
& +\frac{1}{2} \varepsilon_{1}^{-1}(1+n \kappa \delta)^{\varepsilon} c^{n-l}\left\|\Phi_{\kappa}\right\|_{L^{2 l}(\mathscr{T})}^{2 l} .
\end{aligned}
$$

If $l=0$, then the second term in (3.42) equals $\frac{1}{2} \varepsilon_{1}^{-1}(1+n \kappa \delta)^{\varepsilon} c^{n} \ell T$, and we have a bound of the form (3.28). Thus we can assume $1 \leqq l \leqq n-1$. We apply (3.36) to (3.38). Replace $w$ in (3.39) by $w^{2}$ to obtain

$$
c^{n-l}\|\Phi\|_{L^{2 l}(\mathscr{T})}^{2 l} \leqq \varepsilon_{1}^{2}(1+n \delta \kappa)^{-2 \varepsilon}\left\|\Phi_{\kappa}\right\|_{L^{2 n}(\mathscr{T})}^{2 n}+\left(\varepsilon_{1}^{-2(n-1)} c^{n}\right)(1+n \delta \kappa)^{2 \varepsilon(n-1)} \ell T .
$$

Insert (3.43) in (3.42) to yield

$$
c^{(n-l) / 2-j}|W(k, l, j)| \leqq \varepsilon_{1}(1+n \kappa \delta)^{-\varepsilon}\left\|\Phi_{\kappa}\right\|_{L^{2 n}(\mathscr{T})}^{2 n}+\frac{1}{2} \varepsilon_{1}^{-(2 n-1)} c^{n}(1+n \kappa \delta)^{\varepsilon(2 n-1)} \ell T .
$$

Note that if $l \leqq n-2$, then the last term in (3.43) has the factor $(1+n \delta \kappa)^{2 \varepsilon q / p}$ $\leqq(1+n \delta \kappa)^{\varepsilon(n-2)}$ in place of $(1+n \delta \kappa)^{2 \varepsilon(n-1)}$, since now $q / p \leqq(n-2) / 2$. Hence $\varepsilon(2 n-1)$ in $(3.44)$ can be replaced by $\varepsilon(n-1)$. In either case we obtain the bound (3.28) as claimed. This completes the proof of Proposition 3.6.

Completion of the Proof of Lemma 3.5. We remark that $s_{r s j}(\kappa)$ in (3.18) for $\alpha_{n}=1$ satisfies

$$
\left|s_{r s j}(\kappa)\right| \leqq \alpha c_{\kappa}^{r+s}|W(n-2 r-j, n-2 s-j, j)|,
$$

where $\alpha=\sup \alpha_{r s j}$ for $r, s \leqq[(n-2) / 2], j \leqq n$. Using Proposition 3.6.iv, with $\varepsilon_{1}$ replaced by $\varepsilon_{1} / \alpha$, and a new $M$, we infer

$$
\left|s_{r s j}(\kappa)\right| \leqq \varepsilon_{1}(1+n \delta \kappa)^{-\varepsilon}\left\|\Phi_{\kappa}\right\|_{L^{2 n}(\mathscr{T})}^{2 n}+M\left((\delta \kappa)^{\varepsilon(2 n-1)}+1\right) \ln (2+\kappa)^{n} .
$$

This is the bound (3.22) as claimed. 
We have now completed the proof of Proposition 3.4 in case $\mathscr{P}(\xi)=\xi^{n}$. We now return to the general case

$$
\mathscr{P}(\xi)=\xi^{n}+Q(\xi),
$$

where $Q(\xi)$ is a polynomial of degree at most $n-1$. Write

$$
\mathscr{S}^{\mathscr{P}}=\mathscr{S}^{\xi^{n}}+\mathscr{X} .
$$

We claim that given $\varepsilon_{1}>0$, there exists $M<\infty$ such that

$$
\left|\mathscr{X}_{\kappa}\right| \leqq \varepsilon_{1}(1+n \kappa \delta)^{-\varepsilon}\left\|\Phi_{\kappa}\right\|_{L^{2 n}(\mathscr{T})}^{2 n}+M(1+n \kappa \delta)^{\varepsilon(2 n-1)} \ln (2+\kappa)^{n} .
$$

Adding (3.49) to the lower bound on $\mathscr{S}_{\kappa}^{\xi^{n}}$, which is the special case of the proposition proved above, we obtain the proposition for general $\mathscr{P}$.

In order to establish (3.49) we use Proposition 3.6. We expand $\mathscr{X}$ into a sum of terms without normal order, using the following generalization of (3.16), namely

$$
: W(k, l, 0):=\sum_{j=0}^{\min (k, l)} \sum_{\substack{0 \leqq r \leqq[(k-j) / 2] \\ 0 \leqq \leqq \leqq(l-j) / 2]}}(-1)^{r+s+j} c^{r+s} \alpha_{r s j} W(k-2 r-j, l-2 s-j, j),
$$

where

$$
\alpha_{r s j}=\left(\begin{array}{c}
k-j \\
2 r
\end{array}\right)\left(\begin{array}{c}
l-j \\
2 s
\end{array}\right)\left(\begin{array}{l}
k \\
j
\end{array}\right)\left(\begin{array}{l}
l \\
j
\end{array}\right) j !(2 r-1) ! !(2 s-1) ! !
$$

Then

$$
\mathscr{X}_{\kappa}=\sum_{\substack{0 \leqq k, l \leqq n \\ 0 \leqq k+l \leqq 2 n-1}} \bar{\alpha}_{k} \alpha_{l}: W(k, l, 0):
$$

and using (3.51)-(3.53) we have reduced $\mathscr{X}$ to a sum of terms for which Proposition 3.6 gives the desired bound. This completes the proof.

\section{Stability under Perturbations}

In this section we consider two sorts of perturbations of $H$ in Theorem 3.1. First we consider how $H$ varies if we vary the coefficients of the polynomial $\mathscr{P}$ defining $H$. Secondly we show that $H$ remains stable under the addition of a class of polynomials $Q$.

Let $I^{2}(\mathfrak{F})$ denote the ideal of Hilbert Schmidt operators on Fock space. In Theorem 3.1, we show that $H=H(\mathscr{P})$ has a heat kernel $\exp (-T H(\mathscr{P})) \in I^{2}(\mathfrak{F})$. We now consider how the heat kernel varies as the coefficients of $\mathscr{P}$ vary over a bounded set of $\mathbb{C}^{n}$. Let

$$
\mathscr{P}(\xi)=\sum_{j=0}^{n} \alpha_{j} \xi^{j}, \quad \alpha_{j} \in \mathbb{C}, \quad\left|\alpha_{j}\right| \leqq M, \quad\left|\alpha_{n}\right| \geqq \varepsilon>0 .
$$

Theorem 4.1. As $\mathscr{P}$ varies over the set (4.1), the map

$$
\left(\alpha_{1}, \ldots, \alpha_{n}\right) \rightarrow e^{-H(\mathscr{P})}
$$

is continuous from $\mathbb{C}^{n}$ to $I^{2}(\mathfrak{F})$. The continuity is uniform in $\chi$ satisfying (2.5). 
Proof. Let $\mathscr{P}_{1}, \mathscr{P}_{2}$ be two polynomials in the set $(4.1)$. Then writing $H_{1}=H\left(\mathscr{P}_{1}\right)$, etc.,

$$
\left\|e^{-H_{1}}-e^{-H_{2}}\right\|_{I^{2}(\widetilde{\mho})}^{2}=\operatorname{Tr}\left(\left(e^{-H_{1}}-e^{-H_{2}}\right) e^{-H_{1}}\right)-\operatorname{Tr}\left(e^{-H_{2}}\left(e^{-H_{1}} e^{-H_{2}}\right)\right) .
$$

Each term in (4.3) has a functional integral representation. The first term, for example, is

$$
\int\left(e^{-\mathscr{S}_{1}}-e^{-\mathscr{S}_{2}}\right) e^{-\hat{\mathscr{S}}_{1}} d \mu_{\mathrm{C}}
$$

where $d \mu_{C}$ is now the Gaussian measure on $\mathscr{D}^{\prime}(\mathscr{T})$, with $\mathscr{T}=S_{l}^{1} \times S_{2}^{1}$. Furthermore, for $j=1,2$,

and

$$
\mathscr{S}_{j}=\int_{0}^{1}:\left\langle\mathscr{P}_{j}\left(\Phi_{\chi}(\cdot, t)\right), V \mathscr{P}_{j} \Phi_{\chi}(\cdot, t)\right\rangle: d t
$$

$$
\hat{\mathscr{S}}_{j}=\int_{1}^{2}:\left\langle\mathscr{P}_{j}\left(\Phi_{\chi}(\cdot, t)\right), V \mathscr{P}_{j} \Phi_{\chi}(\cdot, t)\right\rangle: d t
$$

We estimate (4.4). The estimate of the other term in (4.3) is similar. Write

$$
e^{-\mathscr{S}_{1}}-e^{-\mathscr{S}_{2}}=\left(\mathscr{S}_{2}-\mathscr{S}_{1}\right) \int_{0}^{1} e^{-\left(\alpha \mathscr{S}_{1}+(1-\alpha) \mathscr{S}_{2}\right)} d \alpha .
$$

Using the Hölder inequality on $L^{2}\left(d \mu_{C}\right)$,

$$
\begin{gathered}
\left|\int\left(e^{-\mathscr{S}_{1}}-e^{-\mathscr{S}_{2}}\right) e^{-\hat{\mathscr{S}}_{1}} d \mu_{C}\right| \\
\leqq\left\|\mathscr{S}_{2}-\mathscr{S}_{1}\right\|_{L^{2}\left(d \mu_{C}\right)} \sup _{0 \leqq \alpha \leqq 1}\left(\int e^{-4\left(\alpha \mathscr{S}_{1}+(1-\alpha) \mathscr{S}_{2}\right)} d \mu_{C}\right)^{1 / 4}\left(\int e^{-4 \hat{\mathscr{Y}}_{1}} d \mu_{C}\right)^{1 / 4} .
\end{gathered}
$$
facts:

The continuity of the right side of $(4.5)$ in $\left(\alpha_{1}, \ldots, \alpha_{n}\right)$ is a consequence of two

(i) The action functions $\mathscr{S}_{1}, \mathscr{S}_{2}, \hat{\mathscr{S}}_{1}, \hat{\mathscr{S}}_{2}$ satisfy the estimates of Proposition 3.3 uniformly for all polynomials $\mathscr{P}$ satisfying (4.1).

(ii) The moment $\left\|\mathscr{S}_{2}-\mathscr{S}_{1}\right\|_{L^{2}\left(\mathrm{~d} \mu_{c}\right)}$ is uniformly continuous in the coefficients $\alpha_{1}, \ldots, \alpha_{n}$ for $\left\{\alpha_{j}\right\}$ satisfying (4.1).

The first fact is an elementary consequence of the proof of Theorem 3.1. It is important that $\alpha_{n}$ is bounded away from zero. The second fact is true by inspection of the integral defining the moment.

The second type of perturbation which does not destroy our stability bound is of the form

$$
H(\mathscr{P}, Q)=H+Q
$$

where $H$ is a Hamiltonian discussed in Theorem 3.1 and where $Q$ is a polynomial in $\varphi$ of degree at most $2 n-1$. More specifically, we take $Q$ to be a sum of terms of the form

$$
\int_{\left(S_{\ell}^{1}\right)^{k}}: \varphi_{\chi}\left(x_{1}\right)^{r_{1}} \ldots \varphi_{\chi}\left(x_{k}\right)^{r_{k}}: f\left(x_{1}, \ldots, x_{k}\right) d x_{1} \ldots d x_{k} .
$$

Here $r_{1}+\cdots+r_{k}=r \leqq 2 n-1$, and $f \in L^{p}$ for $p / r \geqq \max \left(r-r_{i}\right)^{-1}$. Methods similar to the ones used above lead to 
Theorem 4.2. For $H(\mathscr{P}, Q)$ of the form (4.6) with $Q$ a finite sum of terms of the form (4.7), the map

$$
\left\{f_{i}\right\} \rightarrow e^{-H(\mathscr{P}, Q)}
$$

is continuous from $\bigoplus_{i} L^{p_{1}} \rightarrow I^{2}(\mathfrak{F})$. The continuity is uniform for $\chi$ satisfying (2.5).

\section{Families $H(\rho, \mathscr{P}, Q)$}

In this section we investigate a family of Hamiltonians $H(\rho, \mathscr{P})$ with a fixed interaction polynomial $\mathscr{P}$ as we vary the cutoff function $\chi$ and the potential $V$. The parameter $\rho \in(0, \infty)$ will yield a continuous family of heat kernels $\exp (-T H(\rho, \mathscr{P}))$. We study this family and its limit as $\rho \rightarrow \infty$ (the ultraviolet limit in which the cutoff tends to the identity).

5.1. Continuity in $\rho$. We introduce $\rho$ into $V$ by defining

$$
\tilde{V}_{\rho}(p)=\tilde{V}\left(\frac{p}{\rho}\right),
$$

where we assume $V$ to be an $\operatorname{SDI}_{\varepsilon}$ cutoff function. We study the Hamiltonian $H(\rho, \mathscr{P}, Q)$, where we introduce $\rho$ into a Hamiltonian (4.6) $H(\mathscr{P}, Q)$ studied in Sect. 3 (with $Q=0$ ) or in Sect. 4. This Hamiltonian may also depend on a cutoff $\chi$ satisfying (2.5), as introduced in Sects. 3 and 4.

Theorem 5.1. For $\rho$ bounded away from 0 and $\infty$, the map

$$
\rho \rightarrow e^{-H(\rho, \mathscr{P}, Q)}
$$

is continuous as a function from $\mathbb{R}$ to $I^{2}(\mathfrak{F})$. This continuity is uniform in $\chi$ satisfying (2.5).

Proof. The uniformity of the bound on $\operatorname{Tr}\left(e^{-H(\rho, \mathscr{P})}\right)$ follows immediately from the inspection of the proof of Theorem 3.1. Hence the continuity in $\rho$ can be investigated as in the proof of Theorem 4.1. We require continuity of $\left\|S_{\rho}-S_{\rho^{\prime}}\right\|_{L^{2}\left(d \mu_{c}\right)}$. This in turn is an elementary consequence of the convergence of the integrals defining the moment $\left\|S_{\rho}-S_{\rho^{\prime}}\right\|_{L^{2}\left(d \mu_{c}\right)}^{2}$. Each integrand is bounded uniformly by an integrable function for $\rho, \rho^{\prime}$ in a compact interval. Furthermore the integrand vanishes for $\rho=\rho^{\prime}$. Hence by the dominated convergence theorem the norm $\left\|S_{\rho}-S_{\rho^{\prime}}\right\|_{L^{2}\left(d \mu_{c}\right)}$ vanishes as $\left|\rho-\rho^{\prime}\right| \rightarrow 0$.

As a consequence of the uniformity of the continuity in $\chi$, we have

Corollary 5.2. If $\chi_{n}$ is a sequence of cutoffs satisfying (2.5) and $\tilde{\chi}_{n} \rightarrow \tilde{\chi}$ pointwise, then

$$
\left\|\exp \left(-H_{n}(\rho, \mathscr{P}, Q)\right)-\exp (-H(\rho, \mathscr{P}, Q))\right\|_{I^{2}(\mathscr{F})} \rightarrow 0 .
$$

5.2. The Limit $\rho \rightarrow \infty$. The investigation of the $\rho \rightarrow \infty$ limit is also straightforward. In this section we fix $\mathscr{P}$ and investigate the continuity of $\exp (-T H(\rho, \mathscr{P}))$ in $\rho$ and the uniformity of the lower bound as $\rho \rightarrow \infty$. In this limit $\tilde{\chi}_{\rho}(p) \rightarrow 1$ pointwise. The estimates of Sect. 3 are stated in terms of their dependence of a cutoff $\chi$ on $\varphi$ and a potential $V$. In Sect. 3, the estimates are uniform in $\chi$ but depend on $V$. Inspection of the bounds show, however, that the dependence on $\rho$ enters through $\delta$ being replaced by $\delta / \rho$. Thus as $\rho \rightarrow \infty$, the constant $\delta$ in Sect. 3 tends to zero. This improves the lower bound on $\mathscr{S}_{\kappa}$ (Proposition 3.4) and it does 
not affect the bound on $\mu\left\{\left|\mathscr{S}-\mathscr{S}_{\kappa}\right| \geqq 1\right\}$, Proposition 3.3. Hence the bound on $\operatorname{Tr} \exp (-H(\rho, \mathscr{P}))$ is uniform as $\rho \rightarrow \infty$. We thus conclude as above that:

Theorem 5.3. The map (5.1) is also continuous as $\rho \rightarrow \infty$.

Acknowledegement. We thank P. da Veiga for reading the manuscript.

\section{References}

1. Glimm, J., Jaffe, A.: Quantum Physics: A Functional Integral Point of View. 2nd ed. Berlin, Heidelberg, New York: Springer 1987

2. Hoegh-Krøhn, R.: Relativistic Quantum Statistical Mechanics in Two Dimensional SpaceTime. Commun. Math. Phys. 38, 195-224 (1974)

3. Nelson, E.: A Quartic Interaction in Two Dimensions. In: Mathematical Theory of Elementary Particles, Goodman, R., Segal, I. (eds.), Cambridge: MIT Press 1966

Communicated by A. Jaffe 
